\title{
Sediment Transport Dynamics in River Networks Induced by Water Diversion
}

\author{
Xu-Ming Wang, Jie Huo, Rui Hao and Shi-Feng Cao \\ NingXia University \\ P.R.China
}

\section{Introduction}

A river network is a typical completely open system formed by interconnected river channels. The interactions between the imports of a river (water and sediment) and the channel will cause the change of the channel patterns. This is actually a feedback between the erosion and the sedimentation states of the channel via an adjustment of the sedimentcarrying capability of the stream. This feedback mechanism indicates that river network is a self-organized system (Rodriguez-Iturbe, 1997), and some dynamical laws lead its evolution and some statistic laws dominate its steady state (Leopold, 1953; Leopold \& Maddock, 1953; Dodds \& Rothman, 2000). So, river networks have attracted, in decades past, a good much attention of physicists and geophysicists (Banavar, et al., 1997; Manna \& Subramanian, 1996; Manna, 1998; Sinclair \& Ball, 1996; Kramer \& Marder, 1992; Takayasu \& Inaoka, 1996; Maritan, et al., 1996; Caldarelli, et al., 1997; Giacometti, 2000; Somfai \& Sander, 1997; Rinaldo, et al., 1996, and so on). They focused mainly on the distributions of river parameters or the scaling relations between them, as well as the evolutionary mechanism, that is, what creates the distributions and scaling relations?

The pioneering field investigation executed by Leopold revealed that the slope, width and depth of a channel respectively depend on the discharge in power functions (Leopold \& Maddock, 1953). From then, some other scaling relations, in power functions, between river parameters were found (Hack, 1957; Flint, 1974). The theoretical studies for the purpose of getting deeper understandings of the reasons why the nature has selected these laws were conducted by the dynamic modeling based on the local erosion rules (for instance, Banavar, et al. ,1997) or the other considerations, such as erosion process based on the minimum energy dissipation (Sun, 1994; Dhar, 2006), evolution of a quasi-random spanning tree (Manna, 1996), statistical physics method based on the self-similarity theory (Banavar, et al. ,1997), and so on.

Our previous modeling studies were focused on another process, that is, sediment transport in river networks (Wang, et al. 2008; Hao, et al., 2008; Huo, et al., 2009). The core spirit of the models embodies the feedback mechanism between erosion and sedimentation via the adjustment of sediment-carrying capability (SCC) of runoff. A steady state shows scaling law that the quantity of erosion or sedimentation (QES) distributes exponentially along the channel in the downriver direction. The response of a river to the abrupt change of the input shows self-organized and self-adaptive behaviors. The former is represented by opposite variation of the SCC to the QES as the discharge changes, which shows that the response of the river trends to depress the increase of erosion as water flow increases and that of 
sedimentation as water flow decreases, the latter is denoted by the deformation of the channel forced by sediment erosion or sedimentation. Sediment concentration is a key factor that influences the sediment transport dynamics in higher-water seasons for its higher value. A higher sediment concentration implies that the stream will inject more sediment into its downriver neighbouring segment of the channel. It follows that the SCC of the stream flowing in this neighbouring segment will be compelled to improve. In other words, the SCC of a channel will be driven by higher sediment concentration of injecting stream (Huo, et al., 2009). The basic natural process associated with sediment transport in a river network is streams confluence occurs from the top rank segments to the bottom rank ones. The change of water flux caused by the confluence will lead to the deformation of the channel due to erosion or sedimentation. In contrast to the confluence, water diversion is often related to the hydraulic engineering, and therefore of practical significance. It may be similar to confluence that diversion will cause the change of the discharge, and then result in the variation of the QES. In this article, a sediment transport dynamical model, for confluence and/or diversion, is proposed to investigate the influence of water diversion on the mainstream. The model is based on the mechanisms that the SCC of a stream is modified by the QES and driven by the sediment concentration of the injecting streams. As far as these two adjustment mechanisms of SCC are concerned, the former is positive and the latter is passive. The numerical results of this model can simulate the dynamic behavior in real river networks: water diversion can cause sedimentation to increase (for instance, Zhang \& Liang, 1995). The transient dynamics shows some interesting characteristics. (Huo, et al., 2009).

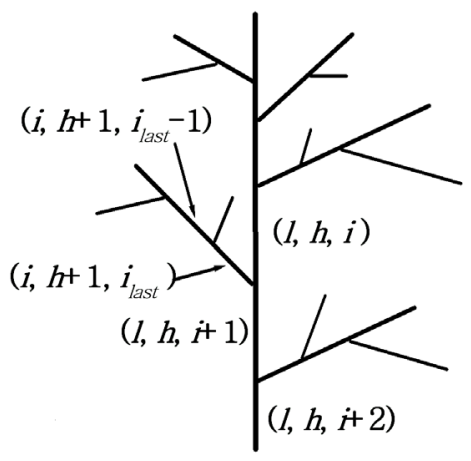

Fig. 1. Schematic drawing of ranks of a river and the segments

In our previous works, we introduced a new ordering scheme of river network (Wang, et al. 2008; Hao, et al., 2008; Huo, et al., 2009). As shown by Fig.1, the rank of a stream can be defined as the times of the confluence while it drains to the sea. If one goes against a stream, say rank $h$, at each of nodes where the confluences occur, the laterally injecting stream belongs to the branches of rank $h+1$, but the non-laterally injecting stream is actually one segment of this stream. So the rank of a stream can be uniquely determined by the times of its laterally injecting confluences as it drains to the sea. And the segments of a stream can be numbered with the times of its non-laterally injecting confluences as it drains to the sea. The first segment is at the upper end, which is actually taken as the source. And then the segment number increases one by one as branches of rank $i+1$ inject into the stream in turn. For convenience, we denote the stream flowing on a channel segment by the segment itself. 
So one segment can be denoted by three characters, for instance, $(l, h, i)$, they represent the ' $i$ th' segment of the ' $l$ th' channel of rank ' $h$ ', respectively.

\section{The model}

As discussed above, a confluence of streams is a fundamental process in natural river networks, while a diversion is often related to the hydraulic engineering. Both of the confluence and diversion will cause change of the discharge, and then result in the variation of the QES. Let's firstly consider the confluence of streams on the segments and diversion on some one segment in a time interval, $t \rightarrow t+d t$, which can be simply denoted by $t$ (one of the discrete time variables, i.e., $t=1,2, \ldots)$. So the confluence or diversion, in the $t$ th time step, can be directly expressed as

$$
Q_{h}^{l}(i+1, t+1)=Q_{h}^{l}(i, t) \pm Q_{h+1}^{i}\left(i_{\text {last }}, t\right) \quad h=0,1,2 \cdots
$$

Where "+" and "-" is applicable to confluence and diversion, respectively. $i, l=1,2, \cdots$, and $i=1$ denotes the source of the stream. The denotation, $i_{\text {last }}$, represents the last segment for a confluence, or the diversion channel for a diversion. This equation indicates that the flux of the stream that is running on segment $i+1$ in time step $t+1, Q_{h}^{l}(i+1, t+1)$, is equal to the flux of the outflows of segment $i$ and the last segment of the $i$ th branch of rank $h+1$ in time step $t$, $Q_{h}^{l}(i, t)$ and $Q_{h}^{i}\left(i_{\text {last }}, t\right)$. It's obvious that $Q_{h}^{l}(i+1, t+1)$ actually denotes the outflow of segment $i+1$ in time step $t+1$, and the outflow of segment $i$ is also the inflow of the segment $i+1$. The transported sediment, $S$, carried by water in a higher-water season (usually has higher sediment concentration, $q$, in compare with that in a lower-water season) not only depends on the flux of the stream but is also driven by the sediment concentration of the injecting stream from its upriver neighbour. The higher the sediment concentration of the stream flowing in segment $i$ is, the more sediment is injecting into segment $i+1$, and the more sediment has to be transported in this segment. Thereupon the following relation can be taken to express the aforementioned passive adjustment mechanism of SCC (Huo, et al. 2009):

$$
S_{h}^{l}(i+1, t)=A_{h}^{l}(i+1, t) Q_{h}^{l}(i+1, t)^{a} q_{h}^{l}(i, t)^{b}
$$

It means that the transported sediment is nonlinearly determined by both stream flow and the sediment concentration of the injecting stream. Where $a$ and $b$ are exponents, which are related to the landform and scale of basin, etc.. $A$ is the so-called sediment-carrying coefficient, which denotes the positive part of the SCC and is adjusted by the undergone erosion or sedimentation state.

Generally, the sediment carried by outflow of a segment may not be equal to that carried by the inflows. So erosion or sedimentation will occur in the segment. Based on the confluence and/or diversion expressed by equation (1) and the definition presented above, the QES in this time step can be determined by equation

$$
\Delta S_{h}^{l}(i+1, t)=S_{h}^{l}(i+1, t)-\left(S\left(_{h}^{l}(i, t) \pm S_{h+1}^{i}\left(i_{\text {last }}, t\right)\right)\right.
$$

Where "+" and "-" is for confluence and diversion, respectively. The relation expresses QES on the $i+1$ th segment in the th time step. $\Delta S_{h}^{l}(i, t)>0$ indicates that the segment is eroded, while $\Delta S_{h}^{l}(i, t)<0$ means that it is deposited. 
The positive adjustment of SCC, $A$, is described as follows:

1. Confluence: As presented in our previous studies (Wang, et al., 2008; Hao, et al., 2008 and Huo, et al., 2009), sediment-carrying coefficient A of segment $i+1$ will increase in time step $t+1$ if it is deposited but decrease if it is eroded in time step $t$ due to a basic fact that the sedimentation implies both of the deposited and suspended sediment particles are relatively smaller, which leads to enhancement of the viscosity intensity of fluid and increase in the friction of river-bed against the flow, so the stream can carry more sediment. On the contrary, the erosion implies the deposited material over the river-bed and the suspended particles in the water become coarser. It causes the friction to reduce, thus the stream can transport lesser sediment. So this adjustment of SCC via the variation of $A$ is conducted by the QES on the segment itself.

In fact, $A$ of the stream is also adjusted by QES on its upriver neighbouring segment in the reversed way of that just mentioned above. If a segment is in scouring state, the increasing sediment may cause $A$ of its downriver neighbouring segment to increase so that the stream can discharge more sediment, while if the segment is in deposited state, the decreasing sediment may let $A$ of the downriver neighbouring segment decrease. Naturally, the QES on the last segment of a higher rank branch may work upon $A$ in the same way.

2. Diversion: diversion differs from confluence mainly in the direction of water flowing, and the influence of it on the sediment transport dynamics is principally determined by the so-called water diversion ratio (WDR) that is defined as

$$
\kappa=Q_{h+1}^{i}\left(i_{\text {last }}, t\right) / Q_{h}^{l}(i, t)
$$

and the corresponding sediment diversion ratio(SDR) that is expressed as

$$
\lambda=S_{h+1}^{i}\left(i_{\text {last }}, t\right) / S_{h}^{l}(i, t) .
$$

An experiential equation obtained by a fitting of the field measurement in the Yellow River may generally describe the relation between $\kappa$ and $\lambda$ (Zhao, et al., 1997), that is, $\lambda=B \kappa^{\alpha} Q_{h}^{l}(i, t)^{\beta}$, which means that the diverted sediment nonlinearly depends on WDR and discharge, so we have

$$
S_{h+1}^{i}\left(i_{\text {last }}, t\right)=B \kappa^{\alpha} Q_{h}^{l}(i, t)^{\beta} S_{h}^{l}(i, t) .
$$

As $\kappa>\lambda$ there should be an inequality, $q_{h+1}^{i}\left(i_{\text {last }}, t\right)<q_{h}^{l}(i, t)$, which indicates that the stream, injecting the main channel after diversion, is of lesser water but more sediment. It may compel the stream to improve the SCC, while $\kappa<\lambda, q_{h+1}^{i}\left(i_{\text {last }}, t\right)>q_{h}^{l}(i, t)$ means that the injecting stream after diversion becomes lesser water and lesser sediment. This may abate the burden of transporting sediment for the stream, so the SCC may decrease. Such adjustment of SCC via sediment concentration is the same of the aforementioned passive one in the case of confluence. Meanwhile, the abrupt change of the sediment concentration induced by water diversion will lead to the rapid response of the channel via variation of QES at once. Then the positive adjustment of SCC conducted by QES works here in the same way of confluence. So the positive adjustment of the SCC, $A$, being fit for the description of confluence and/or diversion, can be expressed as

$$
\begin{aligned}
A_{h}^{l}(i+1, t+1)= & A_{h}^{l}(i+1, t)-k_{1} \Delta S_{h}^{l}(i+1, t) /\left(Q_{h}^{l}(i+1, t+1)^{a} q^{\prime}(t+1)^{b}\right) \\
& -k_{2} \Delta S^{\prime}(t) /\left(Q_{h}^{l}(i+1, t+1)^{a} q^{\prime}(t+1)^{b}\right)
\end{aligned}
$$


Where $k_{1}, k_{2}$ denotes the strength of the positive adjustment of SCC respectively from the QES on segment $i+1$ and that of both the QES on segment $i$ and on the last segment of rank $h+1 . \Delta S^{\prime}(t)$ and $q^{\prime}(t+1)$ in Equ.(7) have different meaning for confluence and diversion. According to the discussions above, equations $q^{\prime}(t+1)=q_{h}^{l}(i, t+1)+q_{h+1}^{i}\left(i_{\text {last }}, t+1\right)$ and $\Delta S^{\prime}(t)=\Delta S_{h}^{l}(i, t)+\Delta S_{h}^{i}\left(i_{\text {last }}, t\right)$ satisfy for confluence. As far as the diversion, if the influence of the state of the diversion channel on the main channel can be neglected, we can draw the conclusion that as soon as the diversion is executed the diverted water drops out of the river network. Therefore, there are relations $q^{\prime}(t+1)=q_{h}^{l}(i+1, t+1)$ and $\Delta S^{\prime}(t)=\Delta S_{h}^{l}(i, t)$. The former implies that the SCC of the stream flowing in the segment below and near to the diversion mouth is actually driven by the sediment concentration of the injecting stream.

In our model, any one upriver segment can be chosen as the source of the stream. The regular or irregular change of the discharge $\xi(t)$ represents rainfall. Rainfall usually results in the fluctuation of sediment concentration $\zeta(t)$, and then we have

$$
\begin{gathered}
Q_{h}^{l}(1, t)=Q_{h 0}+\xi(t), \\
q_{h}^{l}(1, t)=q_{h 0}+\zeta(t), \\
\Delta S_{h}^{l}(1, t)=\Delta S_{h 0}
\end{gathered}
$$

for all possible $h, l$ and $t$. Here we assign a fixed value to $\Delta S_{h 0}$ for simplicity.

The core spirit of our model consists in the description of self-adjustability of a river in the sediment transport process, which is manifested by the positive adjustment of SCC that is expressed by Equ. (7). Similar self-adjustability is often related to the dynamics of a completely open system. The simulation work may not only help us to understand the nature of sediment transport in river networks but also provide us with some illumination for general dynamics of self-adaptive systems else.

When a diversion is executed, there are many factors that influence the dynamics of sediment transport such as WDR, SDR, the discharge and the sediment concentration of the injecting fluid. The interaction of these factors will induce much more complex behaviour of the dynamics, which usually differs from that without diversion. Here we present the discussions of the simplest case, $h=0, l=1$, so the superscript $l$ can be deleted in the aforementioned equations. Please note that in our simulation, the diversion mouth is located at the node that connects the $20^{\text {th }}$ segment and the $21^{\text {st }}$ segment. In our study, the values of the parameters and variables are set as $\Delta S_{0}(i, 1)=15.0, S_{0}(i, 1)=10.0, k_{1}=6.0 \times 10^{4}$, $k_{2}=9.0 \times 10^{5}, A(1, t)=5.7 \times 10^{2}, Q_{1}(i, t)=80.0, q_{1}(i, t)=10.0, \quad a=1.1, \quad b=0.7, \quad \alpha=1.2$, $\beta=-0.2, \quad \mathrm{~B}=8.0$ and $Q_{0}(i, 1)=\operatorname{rand}(i=1 \sim 30,100.0 \sim 150.0)$ (random function, $\operatorname{rand}(\cdot)$, denotes the operation that assigning 30 random numbers, produced between 100.0 and 150.0 , to $\left.Q_{0}(i, 1)\right)$.

\section{Influence of WDR on sediment transport}

As discussed above, the relative distance of the value of $\kappa$ and that of $\lambda$ can influence the state of the main channel via changing the SCC of the stream. Fig. 2 shows this relation as the diversion is executed for 6000 time steps, Fig. 3 (a) presents QES on the segments below the diversion mouth and Fig.3 (b) denotes the corresponding SCC. Fig. 2 indicates that there is a critical value of WDR, $\kappa_{c}=0.051$. As $\kappa<\kappa_{c}, \kappa>\lambda$, the injecting stream of the $21^{\text {st }}$ 
segment will become lesser water and more sediment, which implies that the sedimentation will occur due to the forced increase of SCC. This is the passive adjustment of SCC driven by sediment concentration. In addition, the positive adjustment will lead the dynamics to be more complex, and induce the so-called diversion-sedimentation effect, which means that water diversion can cause sedimentation or cause sedimentation quantity to get more (see Fig. 3 (a)). So one can say it is that the positive and passive adjustments of SCC together determine the dynamic behaviour of the $21^{\text {st }}$ segment and those below it even if for the case of $\kappa>\kappa_{c}, \kappa<\lambda$. In this case, the injecting stream of the $21^{\text {st }}$ segment also has a higher SCC. It is partly shown by Fig. 3 (b), and differs sharply from the other segments in the values of $A_{0}$. It follows that the $21^{\text {st }}$ segment will respond intensely to the change caused by diversion.

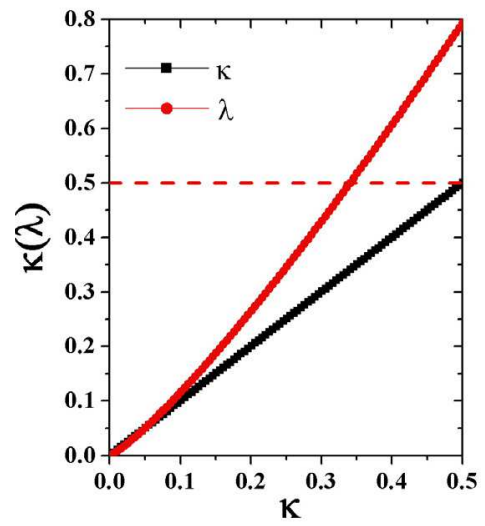

Fig. 2. The relation between WDR and SDR

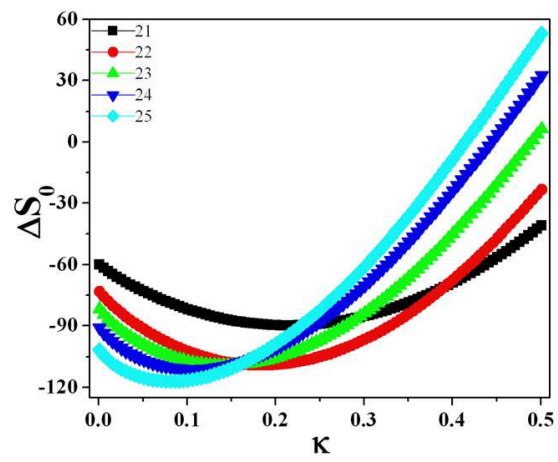

(a)

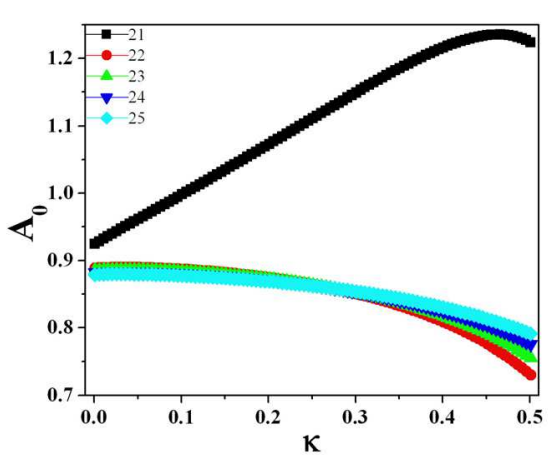

(b)

Fig. 3. Influence of the WDR, $\kappa$, on the dynamics of sediment transport: (a) the QES, $\Delta S$; (b) the positive part of SCC, $A_{0}$

As shown in Fig. 3 (a), there is an another critical value of $\kappa, \kappa_{c}^{\prime}$, for each of the segments below the diversion mouth (the values of $\kappa_{c}^{\prime}$ of the $21^{\text {st }}$ segment, the $22^{\text {nd }}$ segment, ..., is 
$0.216,0.186, \ldots$, respectively) at which the sedimentation quantity on the segments reach the maxima. The maximum indicates that sedimentation will get less with the increase of $\kappa$ as it is beyond $\kappa_{c}^{\prime}$, so one can infer that there is the third critical value of $\kappa$ at which the state of the segments will transform from sedimentation to erosion. It is very interesting that this transform may take place in the segments in turn in the upstream direction. It is noticeable that the maximum of the sedimentation quantity on the segments decreases in downstream direction, and proves that the sedimentation mainly occurs on the segments below and near to the diversion mouth. This behaviour can be attributed to the strong response of the SCC of the $21^{\text {st }}$ segment to the diversion.

To test the aforementioned calculation and get more understandings to the dynamics, we give the QES on the $21^{\text {st }}$ segment obtained analytically. As a matter of convenience, on the $21^{\text {st }}$ segment the increment of the QES, from time step $t$ to time step $t+1$, is taken into account, that is,

$$
\delta \Delta S_{0}(21, t+1)=\Delta S_{0}(21, t+1)-\Delta S_{0}(21, t) .
$$

This equation can be easily expressed as

$$
\delta \Delta S_{0}(21, t+1)=\left(u \kappa^{0.2}-v(1-\kappa)^{0.1}\right) \Delta \kappa-k_{1} \Delta S_{0}(21, t)+k_{2} \Delta S_{0}(20, t) .
$$

Where $u=9.6 Q_{0}^{-0.2}(20, t+1) q_{0}(20, t+1), v=1.1 q_{0}^{0.7}(20, t+1) Q_{0}^{0.4}(20, t+1) A_{0}(21, t)$ and $\Delta \kappa$ is the increment of $\kappa$ in any two neighboring time steps and is assigned a value, 0.0005 . As the parameters and variables take the above values and $Q_{1}(i, t)=295.0$, the comparison between the result obtained analytically and that obtained numerically is shown in Fig.4, and indicates that the result calculated analytically is, on the whole, in agreement with that one obtained numerically. One may note that the value of $\delta \Delta S_{0}(21, t+1)$ changes from the negative to the positive with the increase of $\kappa$. This signifies that the sedimentation quantity on the $21^{\text {st }}$ segment firstly gets more and then becomes less. The variation trends of $\delta \Delta S_{0}(21, t+1)$ is mainly determined by two terms $\kappa^{0.2}$ and $(1-\kappa)^{0.1}$ in Equ. (12), which shows that when $\kappa$ takes a small value $(1-\kappa)^{0.1}$ takes a relative greater value. However, with the increase of $\kappa, \kappa^{0.2}$ increases but $(1-\kappa)^{0.1}$ decreases. It is the reason why the value of $\delta \Delta S_{0}(21, t+1)$ is firstly negative and then become positive, and also the reason why there is the critical WDR, $\kappa_{c}^{\prime}$.

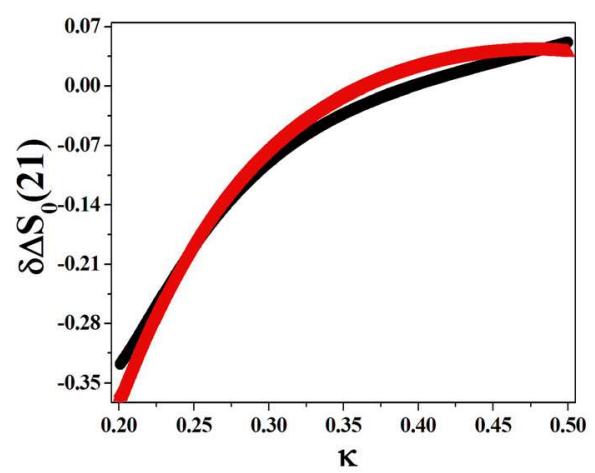

Fig. 4. Comparison of $\delta \Delta S_{0}(21,6000)$ obtained analytically with that obtained numerically 


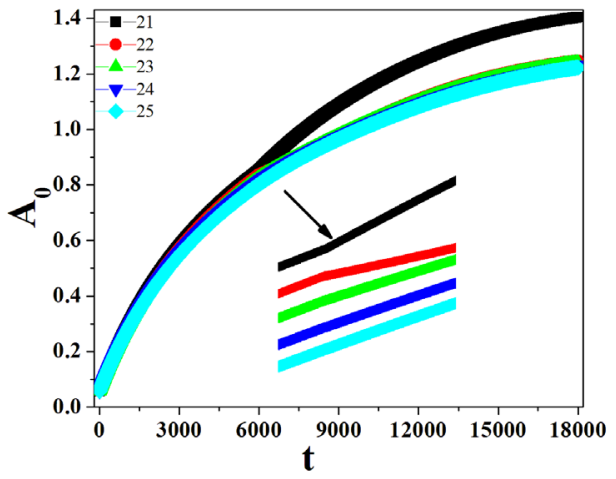

(a)

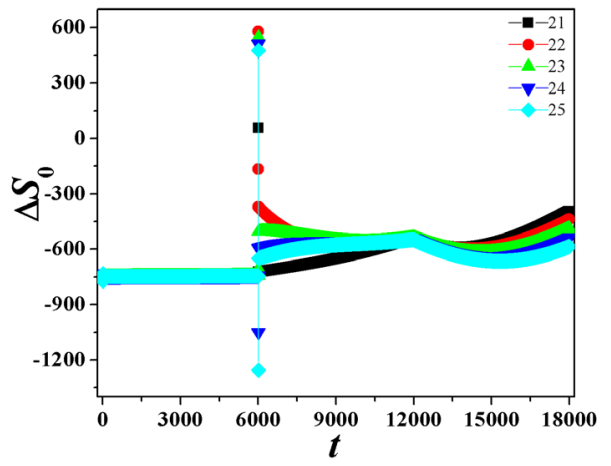

(c)

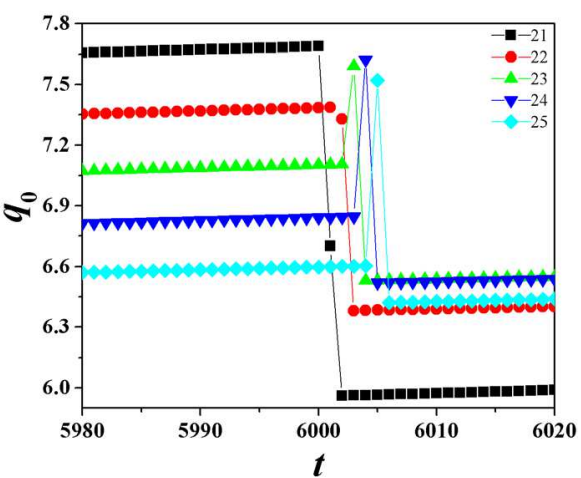

(b)

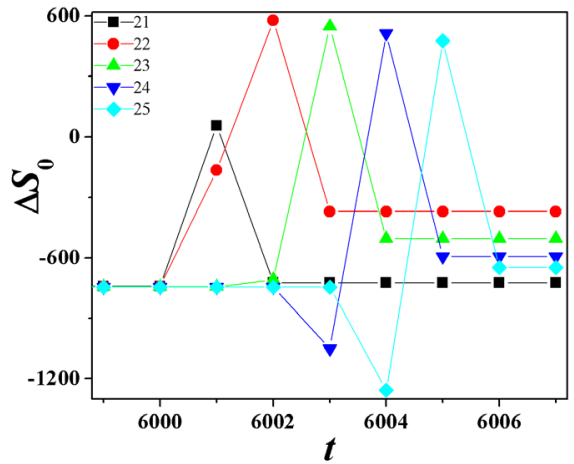

(d)

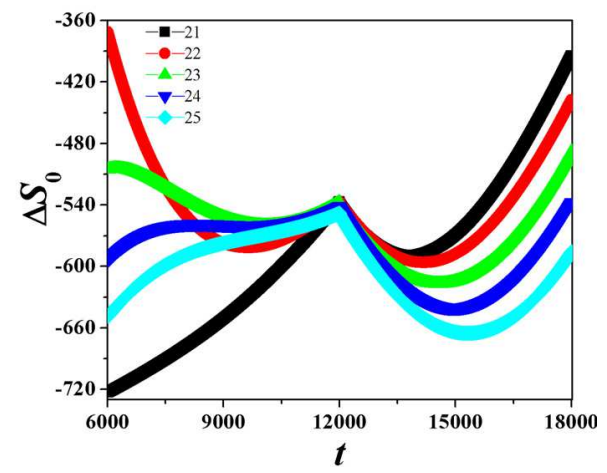

(e)

Fig. 5. Variations of the QES and SCC on the mainstream segments that below the diversion mouth. The details are presented in text. (a) the positive part of SCC, $A_{0}$; (b) sediment concentration, $q_{0}$; (c) QES, $\Delta S_{0}$; (d) a partial magnification of (c) shows initial influence of the steady diversion; (e) another partial magnification of (c) shows the instantaneous process while the discharge of the first rank rivers evenly increase 


\section{Complexity of the sediment transport dynamics induced by diversion}

Since diversion will cause a drastic change of the state, which is described by SCC and QES, of the segment below and near to the diversion mouth, the dynamics of the sediment transport will become more complex. In order to facilitate discussion, we only consider the confluence of the mainstream with the first rank branch rivers. These branch rivers are characterized by $\mathrm{Q}_{1}(i, t)=25.0$ and $\mathrm{q}_{1}(i, t)=30.0$ also for simplicity. The other parameters and variables are set as those in Fig. 3. The simulation is fallen into three phases. The first phase (1-6000 time step) is confluence with fixed imports so as to avoid the unreasonable transience and obtain reasonable SCC and QES for each segment. The second one (600112000 time step) is diversion. It is conducted with the fixed WDR, $\kappa=0.1$, so that we can track the dynamics of the erosion-sedimentation state under steady diversion conditions. After this, the third one runs with the increase of the discharge of each branch of rank 1 , that is, $Q_{1}(i, t)=25.0+\xi(t), \xi(t)=(t-12000) \delta Q_{1}$. Here $\delta Q_{1}=0.0042$, it is the increment of the discharge of the first rank rivers in a time step. In this phase, we mainly investigate the variations of SCC and QES on the segments those below and near to the diversion mouth. Fig. 5 shows these simulation phases, where (a) , (c) denotes the variation of SCC and QES, respectively, in the process; (b) presents the sediment concentration before and after the diversion; (d) is the local magnification of (c), and displays the great changes of the QES caused by the diversion; (e) is also the partial magnification of (c), and demonstrates, in detail, the variation of the QES when it goes to the third phase(the discharge of the main stream and the diverted water increases due to the even increase of the first rank streams) from the second one.

\subsection{Sediment transport dynamics when a steady diversion is conducted}

As shown by Fig.5 (a), the diversion (starts at the fore-end of time step 6001) strengthens the SCC of the stream flowing on the $21^{\text {st }}$ segment immediately. However, the SCC of the stream running on the $22^{\text {nd }}$ segment decreases with a time lag of one step in comparison with the time at which the diversion starts. These changes are denoted by the inflection points on the corresponding curves. Meanwhile, the inflow of the $21^{\text {st }}$ segment decreases suddenly due to the diversion, and as $\lambda>\kappa$, the sediment concentration of the inflow of the $21^{\text {st }}$ segment also decreases suddenly (Fig.5 (b)). The decrease results in the decrease of the sedimentation quantity on this segment (please see Fig.5 (c) and (d)). The decrease of the sediment concentration of the stream flowing on the $21^{\text {st }}$ segment lowers the driving action on the SCC of the stream flowing on the $22^{\text {nd }}$ segments. Therefore, sediment carried by the inflow now gets lesser than that before the diversion is conducted, and then the sedimentation quantity decreases. In time step 6002, the inflow of the 22nd segments decreases since in this time step the outflow of the $21^{\text {st }}$ segment becomes the inflow of the $22^{\text {nd }}$ segment. The sediment carried by these inflow decreases greatly, so a great deal of erosion occurs on this segment. In time step 6003, the stream flowing on this segment becomes steady, it returns to a sedimentation state. The similar changes of the state take place in the segments, $23^{\text {rd }}, 24^{\text {th }}, \ldots$ in turn in the downriver direction. This likes a propagation process in which the amplitude of the change gradually reduces in the downriver direction, and indicates that the influence of the diversion on the segments below the diversion mouth weakens in this direction. It is also demonstrated by Fig.5 (a) that the SCC of the stream, from the $22^{\text {nd }}$ segment to its downriver neighbors, goes down. Fig.5 (e) 
also proves that our simulation is in qualitative accord with the field observation that sedimentation is enhanced, especially on the segments near to the diversion mouth.

One may note if a steady diversion lasts for some time steps, an interesting distribution of the QES will be established. By a careful investigation, we find, via magnifying the part of Fig.5 (a) to which the arrowhead points in the inset, that the SCC of the stream flowing on the $22^{\text {nd }}$ segment decreases suddenly in time step 6003 and then increases gradually. The SCC of the stream flowing on the $23^{\text {rd }}$ segment decreases slightly in time step 6004 . The SCC of the stream of the rest nearly remains changeless. Correspondingly, the variation of erosion-sedimentation state is demonstrated by Fig.5 (e). The monotonously ascending curve means that sedimentation on the $21^{\text {st }}$ segment gets lesser gradually. The sedimentation on the $22^{\text {nd }}$ segment firstly goes down and then goes up. This implies that there is a maximum of the sedimentation quantity on this segment. The curves of QES for the rest of the segments show different changes at first, whereas they tend to be the same of that on the $21^{\text {st }}$ and $22^{\text {nd }}$ segments. So one can infer that on all of the segments, the dynamics of sediment transport is inclined to remove the difference of the QES on the segments, and reach a common state, a so-called nearly erosion-sedimentation equilibrium (NESE) state, at which the state is closed to that one of QES being equal to aero, and the channel keeps unchanged. This is sharply different from that dynamics of sediment transport in river network without diversion, and embodies the complexity of the dynamics caused by diversion.

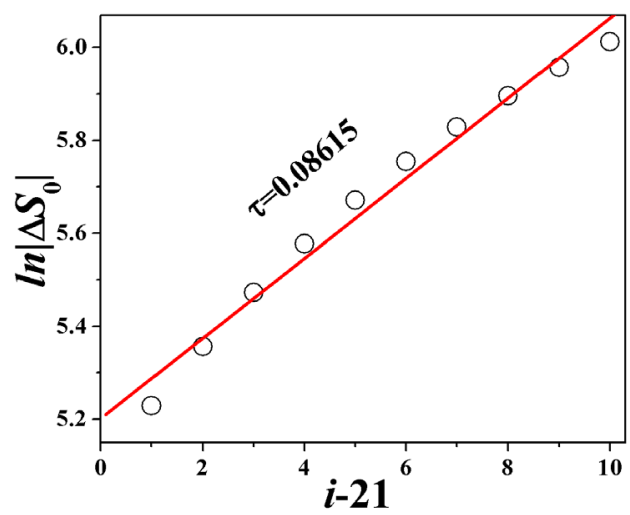

Fig. 6. Scaling law of instantaneous sedimentation distribution on the segments that are below the diversion mouth when they tend to the NESE state

While the dynamics approaches to the NESE, a scaling law that dominates the distribution of the QES can be gradually established, which is described by an exponential function

$$
\left|\Delta S_{0}(i-21, t)\right| \propto \mathrm{e}^{\tau(i-21)} \quad i>21
$$

The scaling function can be obtained by linear fitting in space $\ln \Delta S(i-21, t)$ versus $i-21$, and shown by Fig.6 (presents the calculation at $t=20000$ ). In fact, this scaling function is suitable to the instantaneous distribution, and that the longer the diversion lasts for, the better the distribution accords with an exponential function. The scaling behavior actually embodies the characteristics of the self-adaption and the self-organization. The former refers to the adjustment of the channel via changing the QES to adapt to the import, the latter 
drives at the feedback mechanism between the SCC of the stream and the state of the channel. The former is usually an effect, while the latter is the cause why there exists the result. It is just the feedback mechanism that the behavior shown in Fig.5 from time step 6001 to 6005 when both of the SCC and the QES respond intensely to the sudden change of the imported water and sediment. The intense oscillation between erosion and sedimentation (shown in Fig.5 (d)) denotes this response, the so-called rapid response (Huo, et al., 2009).

\subsection{Influence of the changing discharge on sediment transport dynamics}

As shown in Fig.5 (with fixed WDR), the sudden change of the streams flowing on the first rank branches interrupts the way to the NESE state, and therefore the scaling law expressed by Equ.(13) is destroyed. Since time step 12001, the discharge of the inflow of each mainstream segment increases due to the even increase of the discharge of the first rank rivers. However, the discharge increase of the outflow of these mainstream segments is postponed for one time step in comparison with that of the inflow. These cause the sedimentation quantity on these segments to get more (Fig. 5 (e)). The detail of the response of the QES on these mainstream segments to the discharge increase of the first rank branches can help us to obtain more understandings to the dynamics. For each segment, there is a special section of time series on the curve of $\Delta S_{0}$ near $t=12000$. For a certain segment, it corresponds to the changes resulted from the confluence of the first rank streams, one by one, of which discharge have increased. When the first (top) branch of the first rank that has its discharge increased injects this segment for the first time, the section ends up. The section length of the $21^{\text {st }}$ segment, the $22^{\text {nd }}$ segment ... prolongs for one time step in turn from above to bellow. In the section, the discharge increment of the stream on a segment, for instance, the $21^{\text {st }}$ segment, in each one time step comes from the change of the discharge on the $20^{\text {th }}$ segment after diversion. This increment can be expressed as

$$
\delta Q(21, t+1)=(1-\kappa) \delta Q(20, t)=(1-\kappa)(t-12001) \delta Q_{1} \quad t=12002,12003, \cdots, 12020 .
$$

Since time step 12021, the increment remains changeless. It follows that there is the following relation,

$$
\delta Q(20, t+1)=19 \delta Q_{1} \quad t>12020 .
$$

Obviously, the discharge increment of the stream on the $21^{\text {st }}$ segment reaches the maximum in time step 12020, and then the discharge increases evenly. This change may alter the variation trend of the QES: the increase of sedimentation quantity becomes slower relatively.

One can see from the discussions above, water diversion will give rise to complexity of the sediment transport dynamics, and can further infer a conclusion from this fact that the dynamics will become more complex if a random fluctuation is introduced to mimic a natural rainfall.

Interestedly, if the even increase of the discharge goes on long enough, the transition from sedimentation increasing to sedimentation decreasing will occur in the segments below the diversion mouth in turn from above to below, which indicates that there is an instantaneous maximum of the sedimentation quantity, denoted by $\Delta S_{0 \text { min }}$, for each one of the segments. Fig. 7 suggests the following scaling function:

$$
\left|\Delta S_{0 \min }(i-21)\right| \propto \mathrm{e}^{\gamma(i-21)} \quad i>21 .
$$


This scaling relation reflects the fact that a river network tends to constitute an order to dominate the dynamics in the self-adaption process when the import varies regularly.

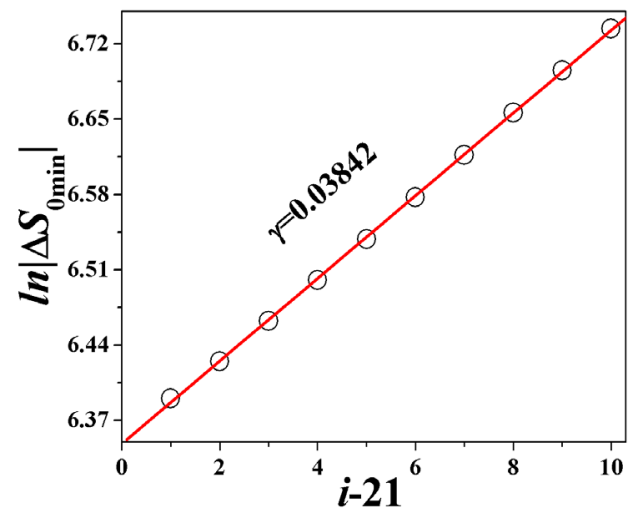

Fig. 7. Scaling law of the maximum sedimentation quantity on the segments that are below the diversion mouth as the flux of water increases linearly

\section{Conclusions and discussions}

In this article, we suggest a dynamical model for sediment transport in river network to study the characteristic of the dynamics induced by water diversion. The model is based on the mechanisms that the SCC of a stream is adjusted by the QES and driven by the sediment concentration of the injecting streams. The numerical results of this model can simulate the dynamical behaviour in real networks: water diversion can cause sedimentation quantity to increase. Some details of the dynamics may help us to understand the nature of river networks and the sediment transport in them.

The transient dynamics shows some interesting characteristics. 1) There is a critical value of WDR at which the state of the segments those are below the diversion mouth will transform from sedimentation getting more to sedimentation getting lesser. 2) Water diversion will cause the dynamics of sediment transport to be more complex. It will lead to the abrupt change of the erosion-sedimentation state. On the segments that are below and near to the diversion mouth sedimentation quantity will oscillate between increase and decrease. This is the rapid response of the channel to the diversion. 3) After the rapid response, if the WDR is fixed the oscillation extent will decay in the downriver direction. The sedimentation quantity on each of the segments will exponentially decreases with time if the steady diversion goes on. This may lead the dynamics to the NESE state near which a scaling law that governs distribution of the QES is established. 4) There is a maximum quantity of sedimentation on each of segments, which is dominated by a scaling law, that is, the maximum quantity of sedimentation depends exponentially on the number of the segments. The aforementioned results may give some enlightenment, especially from the scaling laws. In a river network, the inputs may be random and complex, the outputs (after the transience or in the transient process) may be, however, regular and simple. This dynamic behavior can be attributed to the feedback between the input (stream) and internal factor (the state or the pattern of channel) of the system, and may be common for a kind of completely open system that is endued with self-organizing capacity by the feedback mechanism. 
Of cause, these scaling laws can, generally, not observed in natural river networks since they are destroyed by the large-amplitude random fluctuations in the stream. It follows that the random fluctuation of the discharge caused by rainfalls may play an important role in keeping a relative steadier conformation of the channel.

\section{Acknowledgements}

This study is supported by the National Natural Science Foundation of China under Grant Nos. 10565002 and 10965004, by the Program for New Century Excellent Talents in University of China under Grant No. NCET-06-0914, and by the Ningxia NSF under Grant No. NZ0944.

\section{References}

Banavar, J. R. ; Colaiori, F.; Flammini, A.; Giacometti, A.; Maritan, A. \& Rinaldo, A. (1997). Sculpting of a Fractal River Basin, Phys. Rev. Lett., 78, 23, 4522-4525.

Caldarelli, G.; Giacometti, A.; Maritan, A.; Rodriguez-Iturbe, I. \& Rinaldo, A. (1997). Randomly pinned landscape evolution, Phys. Rev. E, 55, 5, R4865-R4868.

Dhar, D. (2006). Theoretical studies of self-organized criticality, Physica A. 369, 1, 29-70.

Dodds, P. S.\& Rothman, D. H. (2000). Geometry of river networks. I. Scaling, fluctuations, and deviations, Phys Rev. E 63, 016115. and the following papers

Dodds, P. S.\& Rothman, D. H. (2000). Geometry of river networks. II. Distributions of component size and number, Phys Rev. E 63, 016116.

Dodds, P. S.\& Rothman, D. H. (2000). Geometry of River Networks III: Characterization of Component Connectivity, Phys. Rev. E 63, 016117.

Flint, J. J. (1974). Stream gradient as a function of order, magnitude, and discharge, Wat. Resour. Res., 10, 5, 969-973.

Giacometti, A. (2000). Local minimal energy landscapes in river networks, Phys. Rev. E, 62, 5, 6042-6051.

Hack, J. T. (1957). Studies of longitudinal stream profiles in Virginia and Maryland, U. S. Geol. Surv. Prof. Pap. 294B, 45-97.

Hao , R.; Wang , X.-M.; Huo, J. \& Zhang, J.- F. (2008). Simulating sediment transport on river networks, Mod. Phys. Lett. B, 22, 2, 127-137.

Huo, J.; Wang, X.-M.; Hao, R. \& Zhang, J.-F. (2009). Sediment transport dynamics in river networks: A model for higher-water seasons, Lecture Notes of the Institute for Computer Sciences, Social Informatics and Telecommunications Engineering, 4, Part I, 832-840.

Kramer, S. \& Marder, M. (1992). Evolution of river networks, Phys. Rev. Lett., 68, 2, 205-208.

Leopold, L. B. (1953). Downstream change of velocity in rivers, Am. J. Sci., 251, 606.

Leopold, L. B. \& Maddock, T. (1953). The hydraulic geometry of stream channels and some physiographic implications.US Geol. Surv. Prof. pap., 252, 57.

Manna, S. S. (1998) Branched tree structures: From polymers to river networks, Physica A., 254, 1-2, 190-197.

Manna, S. S. \& Subramanian, B. (1996). Quasirandom spanning tree model for the early river network, Phys. Rev. Lett., 76, 18, 3460-3463.

Maritan, A.; Colaiori, F.; Flammini, A.; Cieplak, M. \& Banavar, J. R. (1996). Universality classes of optimal channel networks, Science, 272, 5264, 984-986. 
Maritan, A.; Rinaldo, A.; Rigon, R.; Giacometti, A. \& Rodriguez-Iturbe, I. (1996). Scaling law for river networks, Phys. Rev. E. 53, 2, 1510-1512.

Rinaldo, A.; Maritan, A.; Colaiori, F.; Flammini, A.; Rigon, R.; Rodriguez-Iturbe, I. \& Banavar, J. R. (1996). Thermodynamics of fractal networks, Phys. Rev. Lett. 76, 18, 3364-3367.

Rodriguez-Iturbe, I.; \& Rinaldo, A. (1997). Fractal River Basins:Chance and Self-Organization, Cambridge University Press, Cambridge.

Sinclair, K. \& Ball, R. C. (1996). Mechanism for global optimization of river networks from local erosion rules, Phys. Rev. Lett. 76, 18, 3360-3363.

Somfai, E. \& Sander, L. M. (1997). Scaling and river networks: A Landau theory for erosion, Phys. Rev. E , 56, 1, R5-R8.

Sun, T.; Meakin, P. \& Jøssang, T. (1994). A minimum energy dissipation model for drainage basins that explicitly differentiates between channel networks and hillslopes, Physica A , 210, 1-2, 24-47.

Sun, T.; Meakin, P. \& Jøssang, T. (1994). Minimum energy dissipation model for river basin geometry, Phys. Rev. E, 49, 6, 4865-4872.

Takayasu, H. \& Inaoka, H. (1992). New type of self-organized criticality in a model of erosion, Phys. Rev. Lett., 68, 7, 966-969.

Wang, X.-M.; Hao, R.; Huo, J. \& Zhang, J.-F. (2008). Modeling sediment transport in river networks, Physica A, 387, 25, 6421-6430.

Zhang, D. \& Liang ,Z., (1995). Overview on the relation of water and sediment diversion with erosion/sedimentation of channel of the Lower Yellow River, J. Sediment Res., 2, 32-42. (in Chinese).

Zhao, W.-L. (1997). Sediment in the Yellow River, The Yellow River Water-Conservancy Publishing Company, 7-80621-106-3/TV·82, Zhengzhou, (in Chinese). 


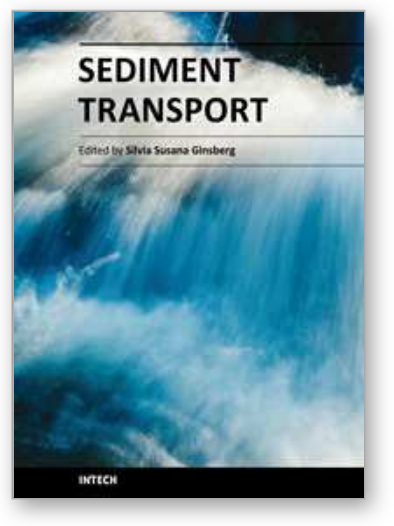

\author{
Sediment Transport \\ Edited by Dr. Silvia Susana Ginsberg
}

ISBN 978-953-307-189-3

Hard cover, 334 pages

Publisher InTech

Published online 26, April, 2011

Published in print edition April, 2011

Sediment transport is a book that covers a wide variety of subject matters. It combines the personal and professional experience of the authors on solid particles transport and related problems, whose expertise is focused in aqueous systems and in laboratory flumes. This includes a series of chapters on hydrodynamics and their relationship with sediment transport and morphological development. The different contributions deal with issues such as the sediment transport modeling; sediment dynamics in stream confluence or river diversion, in meandering channels, at interconnected tidal channels system; changes in sediment transport under fine materials, cohesive materials and ice cover; environmental remediation of contaminated fine sediments. This is an invaluable interdisciplinary textbook and an important contribution to the sediment transport field. I strongly recommend this textbook to those in charge of conducting research on engineering issues or wishing to deal with equally important scientific problems.

\title{
How to reference
}

In order to correctly reference this scholarly work, feel free to copy and paste the following:

Xu-Ming Wang, Jie Huo, Rui Hao and Shi-Feng Cao (2011). Sediment Transport Dynamics in River Networks Induced by Water Diversion, Sediment Transport, Dr. Silvia Susana Ginsberg (Ed.), ISBN: 978-953-307-189-3, InTech, Available from: http://www.intechopen.com/books/sediment-transport/sediment-transport-dynamics-inriver-networks-induced-by-water-diversion

\section{INTECH}

open science | open minds

\section{InTech Europe}

University Campus STeP Ri

Slavka Krautzeka 83/A

51000 Rijeka, Croatia

Phone: +385 (51) 770447

Fax: +385 (51) 686166

www.intechopen.com

\section{InTech China}

Unit 405, Office Block, Hotel Equatorial Shanghai

No.65, Yan An Road (West), Shanghai, 200040, China

中国上海市延安西路65号上海国际贵都大饭店办公楼405单元

Phone: +86-21-62489820

Fax: $+86-21-62489821$ 
(C) 2011 The Author(s). Licensee IntechOpen. This chapter is distributed under the terms of the Creative Commons Attribution-NonCommercialShareAlike-3.0 License, which permits use, distribution and reproduction for non-commercial purposes, provided the original is properly cited and derivative works building on this content are distributed under the same license. 